\title{
Nanoarrays for the generation of complex optical wave-forms
}

\author{
David L. Andrews, ${ }^{\text {a }}$ Mathew D. Williams, ${ }^{\mathrm{a}}$ David S. Bradshaw, ${ }^{\mathrm{a}}$ Ruifeng Lui, ${ }^{\mathrm{b}}$ David B. Phillips, \\ Sonja Franke-Arnold, ${ }^{\mathrm{b}}$ and Miles J. Padgett ${ }^{\mathrm{b}}$ \\ ${ }^{a}$ School of Chemistry, University of East Anglia, Norwich NR4 7TJ, U.K. \\ ${ }^{\mathrm{b}}$ School of Physics and Astronomy, University of Glasgow, Glasgow, G12 8QQ, U.K.
}

\begin{abstract}
Light beams with unusual forms of wavefront offer a host of useful features to extend the repertoire of those developing new optical techniques. Complex, non-uniform wavefront structures offer a wide range of optomechanical applications, from microparticle rotation, traction and sorting, through to contactless microfluidic motors. Beams combining transverse nodal structures with orbital angular momentum, or vector beams with novel polarization profiles, also present new opportunities for imaging and the optical transmission of information, including quantum entanglement effects. Whilst there are numerous well-proven methods for generating light with complex wave-forms, most current methods work on the basis of modifying a conventional Hermite-Gaussian beam, by passage through suitably tailored optical elements. It has generally been considered impossible to directly generate wave-front structured beams either by spontaneous or stimulated emission from individual atoms, ions or molecules. However, newly emerged principles have shown that emitter arrays, cast in an appropriately specified geometry, can overcome the obstacles: one possibility is a construct based on the electronic excitation of nanofabricated circular arrays. Recent experimental work has extended this concept to a phase-imprinted ring of apertures holographically encoded in a diffractive mask, generated by a programmed spatial light modulator. These latest advances are potentially paving the way for creating new sources of structured light.
\end{abstract}

Keywords: complex light, structured light, singular optics, optical angular momentum, Laguerre-Gaussian beam, Bessel beam, optical vortex, quantum optics, chirality, optical manipulation

\section{INTRODUCTION}

Optical beams with intricate wavefront and polarization structures, generically known as 'structured light', are the subject of an escalating volume of research and heightened interest, especially for their applications in the non-contact optical manipulation of matter ${ }^{1}$ (enabling such abilities as microparticle rotation, traction and sorting) and as highcontent optical transmitters of information, ${ }^{2}$ with data transfer speeds of $100 \mathrm{~Tb} \mathrm{~s}^{-1}$ having recently been shown to be achievable in a free-space data link. ${ }^{3}$ Amongst various families of structured light beams exhibiting departures from the conventional forms of wavefront, a particularly interesting group contains those known as optical vortex beams. These are forms of light endowed with orbital angular momenta (OAM), a property due to a helically formed wavefront, completely distinct and separable from photon spin. ${ }^{4}$ Currently, generating such a complex wave-form is usually attained through modification of a conventional Hermite-Gaussian beam, on passage through suitably tailored optical elements. ${ }^{5-8}$ However, principles have now emerged to circumvent such post-hoc arrangements. As will be discussed in section 2, the initial concept was based on excitonic emission from pre-excited nanofabricated circular arrays. Excitonic emission from coupled nanoemitters is a necessity since spontaneous emission from a single emitter cannot produce the required twisted wavefront. This is consistent with the fact that OAM circular dichroism (optical discrimination between left- or right-handed OAM) does not occur in conventional single-photon absorption: ${ }^{9}$ it thus follows from time-reversal arguments that, equally, spontaneous emission from a single source will not release photons with differing quanta of

Metamaterials: Fundamentals and Applications 2014, edited by Nader Engheta, Mikhail A. Noginov, Nikolay I. Zheludev, Proc. of SPIE Vol. 9160, 91601L · (c) 2014 SPIE · CCC code: 0277-786X/14/\$18 · doi: 10.1117/12.2060714 
OAM. The key feature of emission from a ring is that it is possible to secure the interference of fields that differ in phase at their point of origin. Inspired by this theoretical research, subsequent experimental work on the generation of optical beams containing OAM has now proven the possibility of achieving similar results using special holographically encoded apertures, acting as phase-imprinted secondary sources, as detailed in section 3 . This account concludes with a discussion of current directions for further research.

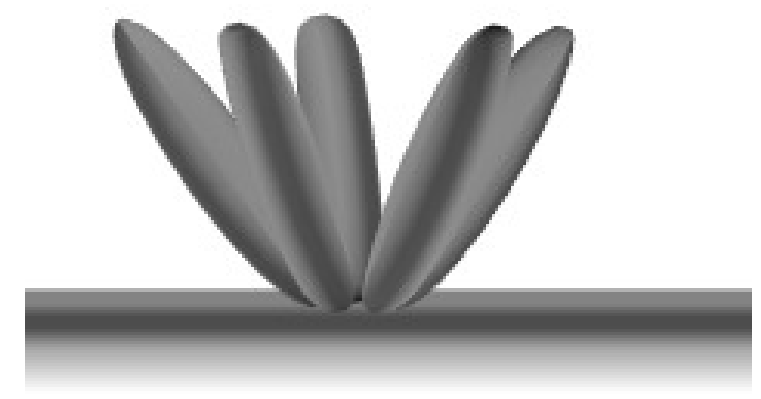

Figure 1. Schematic depiction of a structured array containing five oriented nanoemitters

\section{EMISSION FROM STRUCTURED EMITTER ARRAYS}

The theoretical basis for the direct generation of an optical vortex beam is based on a structured array composed of a number of nanoemitters; ${ }^{10-12}$ for the purposes of this paper, the number chosen is five. By adopting such an array with a geometric configuration shown in Figure 1, it is possible to exploit phase relationships between the quantum amplitudes associated with emission from differing positions. This relationship is satisfied by the formation of an exciton, which arises when electronic excitation is delocalized across identical nanoparticles. Each nanoparticle component is necessarily positioned in an electronically equivalent local environment, and coupled to its neighbors by transition dipole Coulombic interactions of equal magnitude. A circularly symmetric ring fulfils the basic criteria for the array and it is necessary that each emitter has an orientation that is rotated by $2 \pi / 5$ radians with respect to its neighbor (on employing five nanoparticles). Excitation of the structured array to form the exciton usually occurs through direct optical excitation. The primary measure of a vortex beam is though the topological charge, $l$ (signifying an OAM of $l \hbar$ per photon), with positive or negative parity denoting either left- or right-handed gyration respectively. In this five-fold case, optical vortex emission is possible with $l=-2,-1,0,1,2$, as shown in ref. 12 .

The Hamiltonian, $H$, for the 5 -fold nanoemitter array system is based on the following $(5 \times 5)$-matrix:

$$
H=\left(\begin{array}{ccccc}
E_{u} & \boldsymbol{\mu}_{1} \cdot \mathbf{V}_{12} \cdot \mu_{2} & \boldsymbol{\mu}_{1} \cdot \mathbf{V}_{13} \cdot \boldsymbol{\mu}_{3} & \boldsymbol{\mu}_{1} \cdot \mathbf{V}_{14} \cdot \boldsymbol{\mu}_{4} & \boldsymbol{\mu}_{1} \cdot \mathbf{V}_{15} \cdot \boldsymbol{\mu}_{5} \\
\boldsymbol{\mu}_{2} \cdot \mathbf{V}_{21} \cdot \boldsymbol{\mu}_{1} & E_{u} & \boldsymbol{\mu}_{2} \cdot \mathbf{V}_{23} \cdot \mu_{3} & \boldsymbol{\mu}_{2} \cdot \mathbf{V}_{24} \cdot \boldsymbol{\mu}_{4} & \boldsymbol{\mu}_{2} \cdot \mathbf{V}_{25} \cdot \boldsymbol{\mu}_{5} \\
\boldsymbol{\mu}_{3} \cdot \mathbf{V}_{31} \cdot \boldsymbol{\mu}_{1} & \boldsymbol{\mu}_{3} \cdot \mathbf{V}_{32} \cdot \boldsymbol{\mu}_{2} & E_{u} & \boldsymbol{\mu}_{3} \cdot \mathbf{V}_{34} \cdot \boldsymbol{\mu}_{4} & \boldsymbol{\mu}_{3} \cdot \mathbf{V}_{35} \cdot \boldsymbol{\mu}_{5} \\
\boldsymbol{\mu}_{4} \cdot \mathbf{V}_{41} \cdot \boldsymbol{\mu}_{1} & \boldsymbol{\mu}_{4} \cdot \mathbf{V}_{42} \cdot \boldsymbol{\mu}_{2} & \boldsymbol{\mu}_{4} \cdot \mathbf{V}_{43} \cdot \mu_{3} & E_{u} & \boldsymbol{\mu}_{4} \cdot \mathbf{V}_{45} \cdot \boldsymbol{\mu}_{5} \\
\boldsymbol{\mu}_{5} \cdot \mathbf{V}_{51} \cdot \boldsymbol{\mu}_{1} & \boldsymbol{\mu}_{5} \cdot \mathbf{V}_{52} \cdot \boldsymbol{\mu}_{2} & \boldsymbol{\mu}_{5} \cdot \mathbf{V}_{53} \cdot \boldsymbol{\mu}_{3} & \boldsymbol{\mu}_{5} \cdot \mathbf{V}_{54} \cdot \boldsymbol{\mu}_{4} & E_{u}
\end{array}\right),
$$

where $E_{u}$ is the energy of an unperturbed molecule in its excited state $u$. The off-diagonal elements denote emitter pair interactions (as signified by the subscripts), which are cast in the form of inner products of transition dipole moments $\mu_{r}$ and $\mu_{s}$ with a second-rank tensor $V_{r s}$ (representing the coupling between the nanoemitter pair labelled $r$, $s$ ) whose explicit form is given elsewhere. ${ }^{13-14}$ Each of the off-diagonal terms in equation (1) delivers the Coulombic energy, $U$, given by; 


$$
U=\mu_{r \mid i} V_{r s|j| j} \mu_{s \mid j}=\frac{\mu_{r \mid i} \mu_{s \mid j}}{4 \pi \varepsilon_{0} R_{r s}^{3}}\left(\delta_{i j}-3 \hat{R}_{r s \mid i} \hat{R}_{r s \mid j}\right),
$$

where subscripts $i$ and $j$ signify summed Cartesian components, and $R_{r s}$ is the distance between the nanoemitters $r$ and $s$. Setting the transition dipole moments of the five nanoemitters in an equidistant configuration, and fixing their locally equivalent orientations, provides the conditions suitable for the sought optical vortex creation. As a result, all the off-diagonal elements of the matrix of equation (1) return the same value, $U$. Proceeding by diagonalizing the matrix (1), we identify its eigenvectors as the coupled exciton wavefunctions. In terms of individual emitter basis wavefunctions $\xi_{0 / u}^{(i)}(0$ for the ground state, $u$ for the electronically excited state) these state functions are expressed as:

$$
\begin{aligned}
\left|\psi_{ \pm}\right\rangle & =\frac{1}{\sqrt{5}}\left\{\left|\xi_{u}^{(1)} ; \xi_{0}^{(2)} ; \xi_{0}^{(3)} ; \xi_{0}^{(4)} ; \xi_{0}^{(5)}\right\rangle+\varepsilon\left|\xi_{0}^{(1)} ; \xi_{u}^{(2)} ; \xi_{0}^{(3)} ; \xi_{0}^{(4)} ; \xi_{0}^{(5)}\right\rangle+\varepsilon^{*}\left|\xi_{0}^{(1)} ; \xi_{0}^{(2)} ; \xi_{u}^{(3)} ; \xi_{0}^{(4)} ; \xi_{0}^{(5)}\right\rangle\right. \\
& \left.+2 \varepsilon\left|\xi_{0}^{(1)} ; \xi_{0}^{(2)} ; \xi_{0}^{(3)} ; \xi_{u}^{(4)} ; \xi_{0}^{(5)}\right\rangle+2 \varepsilon^{*}\left|\xi_{0}^{(1)} ; \xi_{0}^{(2)} ; \xi_{0}^{(3)} ; \xi_{0}^{(4)} ; \xi_{u}^{(5)}\right\rangle\right\} .
\end{aligned}
$$

Here $\varepsilon=\exp ( \pm 2 \pi \mathrm{i} / 5)$, in which the sign correlates with the subscript on the state wavefunction. Relating to equation (3), the electric field at an arbitrary position $R$ is now expressible as follows, in which $R_{r}$ defines the location with respect to each nanoemitter $r$;

$$
\mathbf{E}_{12345}(\mathbf{R})=-\sum_{r=1,2,3,4,5} \varepsilon^{r-1} \boldsymbol{\mu}_{r} \cdot \mathbf{V}\left(\mathbf{R}_{r}\right)
$$

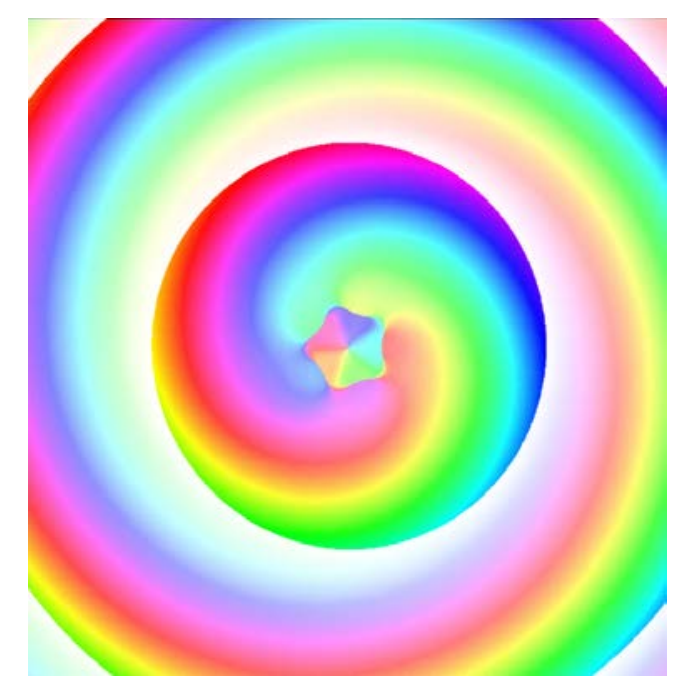

Figure 2. Phase map for the system illustrated by Figure 1 with a topological charge $l=-1$. The phase of the local electric field is represented by color, the local amplitude by color intensity.

Our interest focuses upon the development of the phase for the scalar optical field, which is secured as the complex argument: $\theta(\mathbf{R})=\arg \left\{\mathbf{E}_{12345}(\mathbf{R})\right\}$. Figure 2 exhibits a color-coded representation ${ }^{15}$ of the phase of the emitted near-field, in a plane parallel to the emitter array; the axial phase discontinuity is characteristic of vortex structures. Emission in this direction, perpendicular to the array, will have an opposite sense of wave-front helicity from exciton emission in the opposite direction; the system interchanges chiral character under mirror operation. In practice, the attachment of the array to a surface will favor outward emission. 


\section{HOLOGRAPHICALLY ENCODED APERTURES}

We now describe a novel experimental technique based on the same key principle - namely the imposition of a signature phase relationship between adjacent sources in a ring. These are secondary rather than primary sources, taking the form of a ring of aperture sections within spatial light modulators (SLM), the number of apertures (chosen to be five) corresponding to the number of optically active elements in the previous nanoarray. SLM methods are often used to construct programmable diffractive elements that can generate beams carrying OAM, by combining a spiral phase pattern with a blazed grating to produce a forked hologram that transmits light with OAM into the first order of diffraction pattern. Essentially, the new method exploits this technique with the ray optics principle of apertures acting as sources. The mechanism is angular diffraction, which is well understood, and a rotational analogue to linear diffraction from a linear grating. ${ }^{16}$

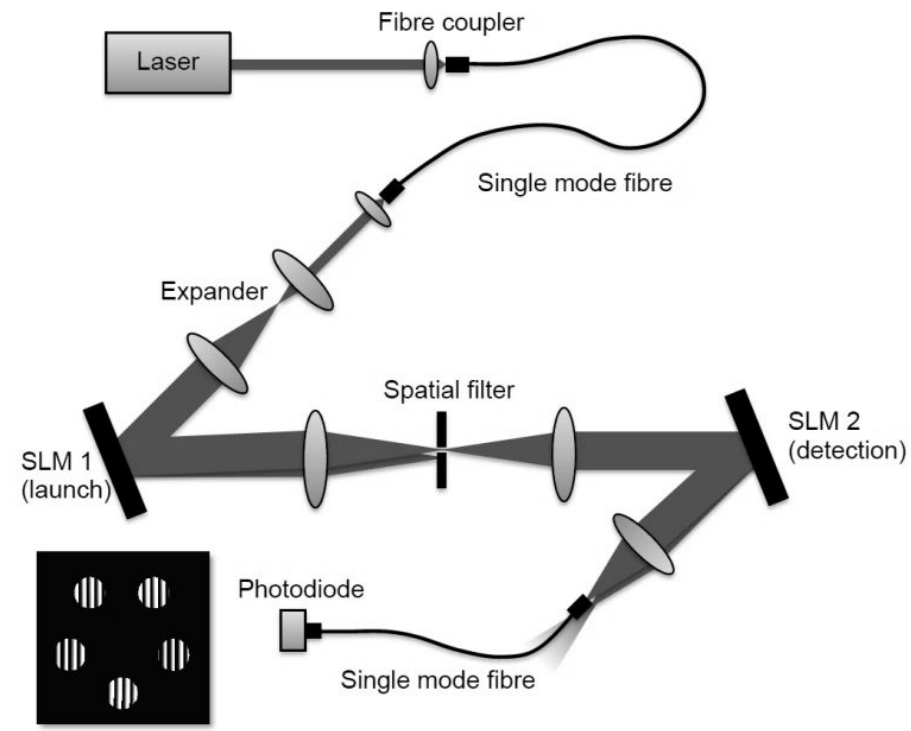

Figure 3. Experimental set up. A $633 \mathrm{~nm}$ wavelength laser beam is coupled into a single mode fiber to generate a pure single mode Gaussian output. This is expanded and reflected from SLM 1 which displays a hologram encoding the distribution and relative phase arrangement of the apertures (five, in the case shown). SLM 2 is used to measure the resulting OAM spectra of the transmitted light, by focusing light of particular OAM modes into the second single mode fiber to the photodetector.

The experimental set-up is illustrated in Figure 3. In detail, a reflection mode spatial light modulator is used to encode a phase pattern of a ring of circular aperture emitters onto a plane wavefront Gaussian beam. ${ }^{17}$ The field is re-imaged through a spatial filter onto a second SLM to measure the OAM spectrum of the resulting light field. This provides a flexible method to investigate an array of aperture configurations. The inset to Figure 3 shows an example of the hologram pattern displayed on SLM 1. Apertures are defined by regions containing diffraction gratings, surrounded by a plane phase background, whilst light reflecting inside an aperture region is diffracted into the first order of the propagating field. The relative phase of light from different apertures is encoded by controlling the relative phase of the diffraction grating. Light reflecting from the SLM outside the apertures remains undiffracted, and therefore goes into the zero order of the propagating field, where it is blocked by the spatial filter centered on the first order beam. SLM 2 is used to measure the OAM spectrum of the transmitted light. A series of forked gratings are consecutively displayed on SLM 2, representing OAM modes $l=-20$ to 20. For each hologram, only light of the conjugate mode is focused into the second single mode fiber and transmitted to the photodiode where its intensity is recorded. For each forked grating 
pattern, light of non-conjugate modes is focused to the characteristic ring shape and therefore does not couple into the photodetector. Undiffracted light also bypasses the photodiode as shown. The relative phase between adjacent apertures is $\delta \phi=2 \pi m / n$, in which $n$ is the number of apertures around the ring and the integer $m$ describes the total phase difference (necessarily not congruent modulo $n$, in the number theory sense, in order that the phase progression has the sought helical character). For example, if $m=2$ and $n=3$ then the total phase difference around the ring is $4 \pi$, and the phase step between adjacent apertures is $\delta \phi=4 \pi / 3$.

Figure 4 exhibits the first results for a typical case, where a ring of five circular apertures on the SLM is phase-imprinted to deliver an output with $m=-1$. The photodiode background signal has been subtracted from the measured signal, which has then been normalized by setting the dominant peak to a value of 1 . Notably, and in contrast to the physical array method, the transmitted field contains other topological charge components, although dominated by the peak associated with at $l=-1$.

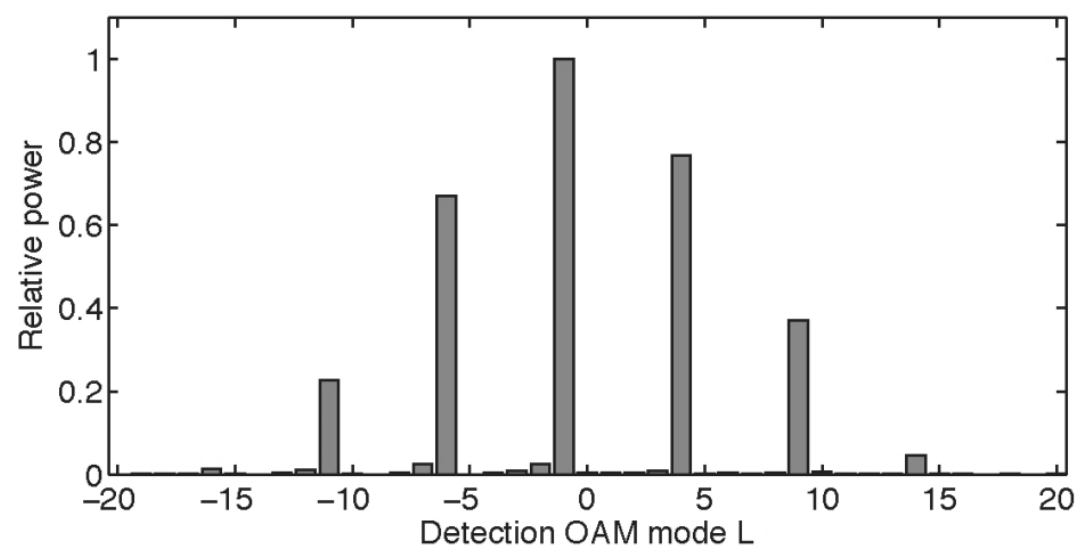

Figure 4: OAM spectra of light generated by a ring of five circular apertures, phase-imprinted to deliver $m=-1$. As a result, the peaks in the OAM spectrum are separated by five modes, i.e. a relative phase difference of $2 \pi / 5$ between neighboring apertures, the dominant peak in the spectrum being found at $l=-1$.

\section{CONCLUSION}

In summary, we have described novel array-based methods for generating light bearing OAM - one, a theory involving real (dipole) emitters, and the other a subsequently developed experimental method using virtual (aperture) emitters. Both systems are based on a ring of emitters with a relative phase difference applied to them. Following the theoretical prediction of the spontaneous emission of a vortex beam, the experimental results represent initial progress towards the realization of such emission. The promising results suggest that these developments might signify the first steps towards a new kind of source for optical vortex light.

\section{ACKNOWLEDGEMENTS}

The authors would like to thank the University of East Anglia and Leverhulme Trust for funding this research. 


\section{REFERENCES}

[1] Simpson, N. B., Dholakia, K., Allen, L. and Padgett, M. J., "Mechanical equivalence of spin and orbital angular momentum of light: An optical spanner,” Opt. Lett. 22, 52-54 (1997).

[2] Gibson, G., Courtial, J., Padgett, M. J., Vasnetsov, M., Pas'ko, V., Barnett, S. M. and Franke-Arnold, S., "Freespace information transfer using light beams carrying orbital angular momentum," Opt. Express 12, 5448-5456 (2004).

[3] Huang, H., Xie, G., Yan, Y., Ahmed, N., Ren, Y., Yue, Y., Rogawski, D., Willner, M. J., Erkmen, B. I., Birnbaum, K. M., Dolinar, S. J., Lavery, M. P. J., Padgett, M. J., Tur, M. and Willner, A. E., “100 Tbit/s freespace data link enabled by three-dimensional multiplexing of orbital angular momentum, polarization, and wavelength,” Opt. Lett. 39, 197-200 (2014).

[4] Bialynicki-Birula, I. and Bialynicka-Birula, Z., "Canonical separation of angular momentum of light into its orbital and spin parts,” J. Opt. 13, 064014 (2011).

[5] Heckenberg, N. R., Mcduff, R., Smith, C. P. and White, A. G., "Generation of optical-phase singularities by computer-generated holograms,” Opt. Lett. 17, 221-223 (1992).

[6] Beijersbergen, M. W., Coerwinkel, R. P. C., Kristensen, M. and Woerdman, J. P., "Helical-wave-front laserbeams produced with a spiral phaseplate,” Opt. Commun. 112, 321-327 (1994).

[7] Marrucci, L., Manzo, C. and Paparo, D., "Optical spin-to-orbital angular momentum conversion in inhomogeneous anisotropic media,” Phys. Rev. Lett. 96, 163905 (2006).

[8] Ostrovsky, A. S., Rickenstorff-Parrao, C. and Arrizón, V., "Generation of the "perfect" optical vortex using a liquid-crystal spatial light modulator,” Opt. Lett. 38, 534-536 (2013).

[9] Andrews, D. L., Dávila Romero, L. C. and Babiker, M., "On optical vortex interactions with chiral matter,” Opt. Commun. 237, 133-139 (2004).

[10] Coles, M. M., Williams, M. D., Saadi, K., Bradshaw, D. S. and Andrews, D. L., "Chiral nanoemitter array: A launchpad for optical vortices,” Laser \& Photon. Rev. 7, 1088-1092 (2013).

[11] Williams, M. D., Coles, M. M., Saadi, K., Bradshaw, D. S. and Andrews, D. L., "Optical vortex generation from molecular chromophore arrays,” Phys. Rev. Lett. 111, 153603 (2013).

[12] Williams, M. D., Coles, M. M., Bradshaw, D. S. and Andrews, D. L., "Direct generation of optical vortices," Phys. Rev. A 89, 033837 (2014).

[13] Daniels, G. J., Jenkins, R. D., Bradshaw, D. S. and Andrews, D. L., "Resonance energy transfer: The unified theory revisited,” J. Chem. Phys. 119, 2264-2274 (2003).

[14] Andrews, D. L. and Bradshaw, D. S., "Virtual photons, dipole fields and energy transfer: a quantum electrodynamical approach,” Eur. J. Phys. 25, 845-858 (2004).

[15] Leach, J., Dennis, M. R., Courtial, J. and Padgett, M. J., “Vortex knots in light,” New J. Phys. 7, 55 (2005).

[16] Jack, B., Padgett, M. J. and Franke-Arnold, S., “Angular diffraction,” New J. Phys. 10, 103013 (2008).

[17] Berkhout, G. C. and Beijersbergen, M. W., "Method for probing the orbital angular momentum of optical vortices in electromagnetic waves from astronomical objects,” Phys. Rev. Lett. 101, 100801 (2008).

*david.andrews@physics.org 